\title{
PRESERVATION OF NORMAL HUMAN PLASMA IN THE LIQUID STATE. II. COMPARATIVE IN VITRO STUDIES ON THE PHYSIOLOGIC ACTIVITY OF LABILE CONSTITUENTS OF LIQUID AND FROZEN PLASMA 1,2
}

\author{
By F. H. L. TAYLOR, EUGENE L. LOZNER,? C. S. DAVIDSON, H. J. TAGNON, AND \\ LLOYD R. NEWHOUSER," WITH THE TECHNICAL ASSISTANCE OF \\ HARRIET MACDONALD AND MARGARET A. ADAMS \\ (From the Thorndike Memorial Laboratory, Second and Fourth Medical Services (Harvard) of the \\ Boston City Hospital; the Department of Medicine, Harvard Medical School, Boston, Massa- \\ chusetts, the Naval Medical Research Institute and the Naval Medical School, National \\ Naval Medical Center, Bethesda, Maryland)
}

(Received for publication November 29, 1943)

The therapeutic use of human plasma is now well established (1 to 3 ). It is available in three preparations, liquid, frozen, and desiccated from the frozen state. The purpose of the present study is an evaluation of liquid and frozen plasma from the standpoint of the stability of certain constituents having important physiological activity.

\section{METHOD}

For the comparative study of liquid and frozen plasma, 3 fresh pools of plasma were prepared by the Blood Plasma Department of the U. S. Naval Medical School, Washington, D. C. One half of each pool was transferred to small containers holding $200 \mathrm{ml}$. of citrated plasma and immediately frozen at $-30^{\circ} \mathrm{C}$. The other half was kept in similar containers in the liquid state at room temperature. At predetermined intervals, a liquid sample and its duplicate frozen sample were shipped to Boston for study. The frozen sample was preserved in this state by shipment in solid carbon dioxide. The usual preparation of the plasma consisted of drawing blood into $35 \mathrm{ml}$. of 2.5 per cent sodium citrate to make a total volume of $300 \mathrm{ml}$. Thus, a 0.31 per cent sodium citrate concentration was obtained. Eighteen hundred $\mathrm{ml}$. of plasma from such bleedings were aspirated after centrifuging into $200 \mathrm{ml}$. of 50 per cent glucose to give a volume of $2000 \mathrm{ml}$. This pool was then subdivided as given above. In one instance,

1 The work described in this paper was done, in part, under a contract, recommended by the Committee on Medical Research, between the Office of Scientific Research and Development and Harvard University.

2 This article has been released for publication by the Division of Publications of the Bureau of Medicine and Surgery of the U. S. Navy. The opinions and views set forth in this article are those of the writers and are not to be considered as reflecting the policies of the Navy Department.

'Lieutenant, Medical Corps, United States Naval Reserve.

4 Captain, Medical Corps, United States Navy. the glucose was omitted to determine if the addition of this substance had any effect on the stability of the labile constituents.

No contamination of any of the samples was found on examination in Boston.

Each plasma was observed for its prothrombin, complement, non-protein nitrogen, total protein, albumin, globulin, fibrinogen, and free hemoglobin content, together with studies on its anti-hemophilic (plasma thromboplastin) properties, recalcification time, and $\mathrm{pH}$. Changes in these various factors were measured over a period of 6 months.

\section{HEMATOLOGICAL STUDIES}

Platelet counts were performed by a direct method using a modified Rees-Ecker diluting solution (4). Normal values by this method vary between 200,000 and 400,000 platelets per c. mm. of whole blood.

Prothrombin concentration was estimated by determining the "prothrombin time" by a modification (5) of Quick's method (6). A "lyophilized" rabbit brain preparation (7) was used as the source of thromboplastin. Using this method, a "prothrombin time" of 25 seconds corresponds to a normal prothrombin concentration. From 80 to 100 per cent of the normal prothrombin concentration was considered to indicate no loss of prothrombin. It has previously been determined that dilution of plasma, to the extent obtained in the preparation of the plasma pools, did not alter the prothrombin concentration within the limits of experimental error.

Determination of anti-hemophilic property. The antihemophilic property of plasma was determined by comparing the coagulation time of $2 \mathrm{ml}$. of blood from a hemophilic subject, with the coagulation time of $2 \mathrm{ml}$. of the same blood to which $0.1 \mathrm{ml}$. of the plasma to be tested had been added. Coagulation times were performed by a modified (8) Lee and White method (9).

The same hemophilic subject was used for testing all samples of plasma. The coagulation time of this particular subject usually ranged between 30 and 60 minutes. A plasma containing a high concentration of anti-hemophilic factor reduced the coagulation time of this individual to a range between 10 and 15 minutes. 
The coagulation time of normal blood by the same method was 5 to 10 minutes.

Recalcification time. The recalcification time was determined by observing the time required for $1 \mathrm{ml}$. of plasma to clot on the addition of $0.2 \mathrm{ml}$. of 0.25 per cent calcium chloride. By this method, normal undiluted blood plasma will coagulate in 6 to 12 minutes.

Determination of plasma hemoglobin. The quantitative estimation of plasma hemoglobin was determined by the benzidine method of Bing and Baker $(10,11)$, modified by Shen and Ham (12).

Normal plasmas should show less than $10 \mathrm{mgm}$. of hemoglobin per $100 \mathrm{ml}$. of plasma by this method.

\section{BACTERIOLOGICAL STUDIES}

Culture.

(1) Plasmas were cultured aerobically in blood broth and on blood agar.

(2) Plasmas were also cultured anaerobically by the following method: $0.5 \mathrm{ml}$. of plasma was added to a tube of blood broth. The cotton plug in the tube was pushed down and a snug-fitting pledget of absorbent cotton then plugged in until it touched the regular stopper. Pyrogallic acid and sodium hydroxide were added above the pledget; the tube closed with a tight-fitting rubber stopper; and the culture incubated at $37^{\circ} \mathrm{C}$.

Titration of complement. The complement titer was determined by observing the smallest amount of plasma which completely hemolyzed a 2 per cent suspension of sheep cells in the presence of 2 units of standard amboceptor. This amount of plasma was then considered to contain one unit of complement. The titer was reported as the number of such units per $1 \mathrm{ml}$. of the plasma.

Normal plasmas contained 5 to 15 units per ml., with the average varying from 10 to 15 units per $\mathrm{ml}$.

\section{CHEMICAL STUDIES}

Estimation of the plasma $\mathrm{pH}$ was made by the glass electrode technique; non-protein nitrogen, by the method of Folin (13); total protein and its partition by a modification of the method of Howe (14). Fibrinogen was determined by a modification of the method of Cullen and Van Slyke (15). Normal values obtained by these methods are as follows: Non-protein nitrogen, 15 to 25 mgm.; total protein, 5.6 to 7.8 grams; albumin, 3.8 to 4.5 grams; globulin, 1.3 to 2.5 grams; and fibrinogen, 190 to $330 \mathrm{mgm}$. per $100 \mathrm{ml}$. of plasma. It should be noted that when fibrinogen was not determinable by the recalcification method, an attempt was made to obtain approximate values by the use of thrombin (16).

\section{EXPERIMENTAL RESULTS}

\section{Plasma proteins}

Over a period of 6 months, the changes in total plasma proteins in both liquid and frozen plasma were not remarkable. No material changes were found in the distribution of albumin or globulin. Fibrinogen, when it was possible to determine it, was present in normal amounts. As is shown in the protocol, fibrinogen could not be determined in the older liquid plasmas, due to failure of the coagulation mechanism. The clotting properties, when thrombin was used, were likewise impaired in some instances.

The non-protein nitrogen of the liquid or frozen plasma did not increase during a period of 6 months. These findings would indicate that both liquid and frozen plasma retained their full values as a source of plasma protein and had lost none of their effectiveness as blood substitutes from the thermodynamic standpoint.

\section{Prothrombin}

There was a marked difference between the prothrombin content of liquid and frozen plasma. Within 6 months, the prothrombin content of liquid plasma fell to less than 2 per cent of its original concentration. Frozen plasma still contained over 40 per cent of its prothrombin content at that time. In 2 instances, where the frozen plasma was thawed slowly over a period of 24 hours, the prothrombin content was reduced to 28 per cent of normal in contrast to plasma thawed rapidly where the prothrombin content was higher. It appears, therefore, that plasma stored in liquid form, for periods as long as 6 months, contains little or no prothrombin, while frozen plasma contains effective amounts of this constituent. Furthermore, it appears that rapid thawing of the frozen plasma is essential if the effective prothrombin content is to be maintained.

Glucose was added to the pooled plasma in 2 of the pools and omitted in one. The presence or absence of the glucose in no way altered the state of preservation of prothrombin.

\section{Anti-hemophilic activity}

The anti-hemophilic component of plasma, "globulin substance" or "plasma thromboplastin" of Howell (17), was remarkably well preserved in both liquid and frozen plasma. In fact, at the end of 6 months, when prothrombin was essentially absent in the liquid plasma, full potency of globulin substance appeared to be present. 


\begin{tabular}{|c|c|c|c|c|c|c|c|c|c|c|c|c|c|c|c|c|c|c|c|c|}
\hline \multirow{4}{*}{ 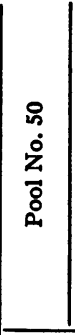 } & \multirow{4}{*}{ 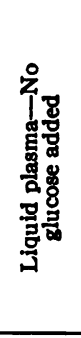 } & 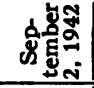 & in & $\underset{\infty}{\infty}$ & 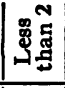 & กั & 芫苍 & 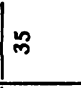 & $=$ & & $\stackrel{0}{\infty}$ & $\mid$\begin{tabular}{|}
$*$ \\
$m$
\end{tabular} & 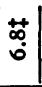 & 菒 & $\underset{+}{+}$ & 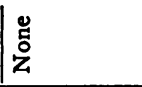 & 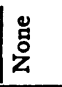 & $\dot{n}$ & & \\
\hline & & 总羊 & 0 & $\div$ & $\approx$ & กี & 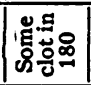 & 3 & 웅 & $\left|\begin{array}{l}8 \\
8 \\
\infty\end{array}\right|$ & $\stackrel{n}{\sim}$ & 芦 & $\begin{array}{l}0 \\
\vdots \\
\vdots \\
0\end{array}$ & 蔦 & 苔 & 荵 & 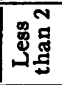 & $\check{z}$ & & \\
\hline & & 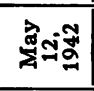 & + & 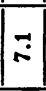 & 요 & ชั & 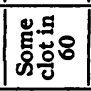 & $\check{m}$ & $\dddot{m}$ & $\begin{array}{l}8 \\
8 \\
-1\end{array}$ & & 营 & 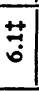 & 莒 & 苔 & 荵 & 웅 & ¿্ّ & & \\
\hline & & 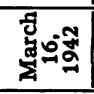 & -1 & & 앙 & ช̃ & $F$ & 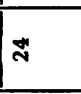 & 웅 & $\begin{array}{l}8 \\
0 \\
\\
-1\end{array}$ & $\stackrel{\infty}{\sim}$ & 营 & 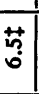 & 菏 & 薃 & 蒙 & $\simeq$ & ż & & \\
\hline \multirow{8}{*}{ 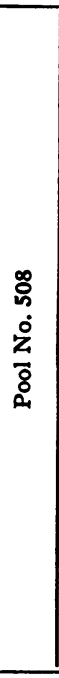 } & \multirow{4}{*}{ 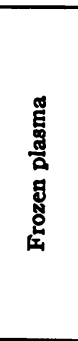 } & 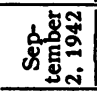 & $\infty$ & $\vec{\infty}$ & q & กี & 웅 & $m$ & $=$ & & $\ddot{m}$ & 志 & 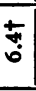 & 索 & 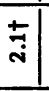 & 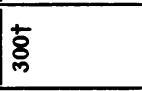 & $\stackrel{2}{2}$ & ż & $\bar{N}$ & \\
\hline & & 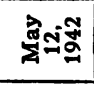 & - & $\stackrel{n}{\sim}$ & $\ddot{\circ}$ & กี & 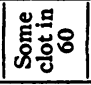 & $\ddot{m}$ & $\nexists$ & $\begin{array}{l}8 \\
+\end{array}$ & & 売 & స్ & 5 & $\mid$ & 声 & $\stackrel{2}{2}$ & 峁 & $\bar{N}$ & \\
\hline & & 营琣 & 0 & 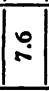 & 3 & ชั & $=$ & $F$ & $\approx$ & $\mid \begin{array}{l}8 \\
8 \\
-1\end{array}$ & 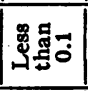 & 总 & 京 & \pm & $\begin{array}{l}\text { ț } \\
\dot{i}\end{array}$ & 䇏 & $\stackrel{2}{\circ}$ & ż & 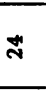 & \\
\hline & & 苟的 & in & & 部 & ชุ & $\stackrel{\infty}{=}$ & 嘿 & $\cong$ & $\mid \begin{array}{l}8 \\
8 \\
-5\end{array}$ & & 孛 & $\begin{array}{c} \pm \\
\vdots \\
0\end{array}$ & $\begin{array}{c}\vdots \\
+ \\
+\end{array}$ & 志 & 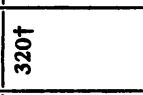 & & ڤ্ & $\approx$ & \\
\hline & \multirow{4}{*}{ 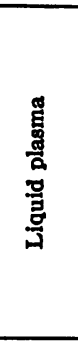 } & 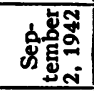 & & $\vec{\infty}$ & 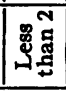 & ชี & $\stackrel{\circ}{2}$ ơ & m & $\Rightarrow$ & & $\stackrel{i}{i}$ & 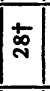 & ț. & 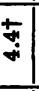 & 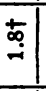 & 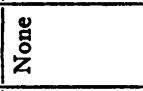 & 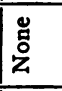 & ¿ّ & & \\
\hline & & 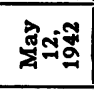 & $m$ & $\stackrel{m}{\sim}$ & 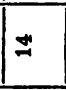 & กั & 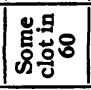 & 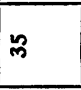 & $\approx$ & \begin{tabular}{|l|} 
\\
0 \\
0
\end{tabular} & & 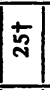 & t⿱ & 志 & \pm & ন & 윽 & ż & & \\
\hline & & 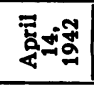 & $N$ & $\stackrel{\square}{\because}$ & 웅 & กี & 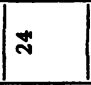 & $F$ & $\nexists$ & \begin{tabular}{|l|} 
\\
\\
-1
\end{tabular} & 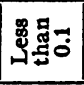 & 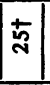 & 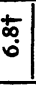 & 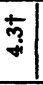 & ก) & ลे & m & ż & & \\
\hline & & 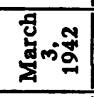 & - & & 8 & กุ. & $\neq$ & m & 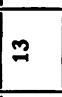 & \begin{tabular}{|l|}
8 \\
8 \\
$\infty$
\end{tabular} & & 志 & 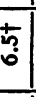 & 京 & 荢 & 索 & in & ż & & \\
\hline \multirow{8}{*}{$\begin{array}{l}8 \\
\dot{0} \\
z \\
\overline{8} \\
0\end{array}$} & \multirow{4}{*}{ 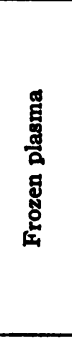 } & 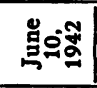 & & $\stackrel{0}{*}$ & 2 & วั & iి & 2 & $=$ & $\mid$ & $\infty_{\infty}^{\circ}$ & 志 & $\begin{array}{l}+ \\
\vdots \\
0\end{array}$ & $\begin{array}{l}+ \\
+ \\
+\end{array}$ & $\stackrel{+}{a}$ & t) & $\dddot{2}$ & ¿ّ & n & 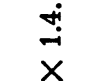 \\
\hline & & 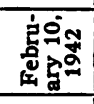 & - & & 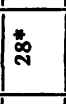 & กั & $\exists$ & $\infty_{\infty}^{\infty}$ & $m$ & 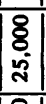 & & $\mid \begin{array}{c}+ \\
\stackrel{\infty}{*}\end{array}$ & $\begin{array}{l}+ \\
\vdots \\
0 \\
0\end{array}$ & 莗 & స్ & 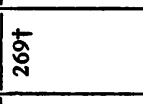 & $\mp$ & 通 & $\nexists$ & $\hat{\text { ș }}$ \\
\hline & & 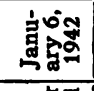 & 0 & & 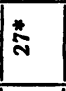 & ชุ & $\neq$ & m. & $\approx$ & \begin{tabular}{|l|}
8 \\
0 \\
$\infty$ \\
\multirow{3}{*}{}
\end{tabular} & & 声 & $\begin{array}{l}+ \\
\vdots \\
0\end{array}$ & $\stackrel{\square}{+}$ & 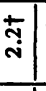 & t⿱艹 & $\approx$ & זّ & 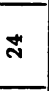 & \\
\hline & & d产 & in & & 8 & & & i & $\approx$ & \begin{tabular}{|l|}
8 \\
0 \\
4
\end{tabular} & & $\mathbf{t}$ & 索 & 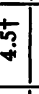 & ث. & 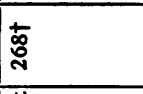 & & z⿺ & - & \\
\hline & \multirow{4}{*}{ 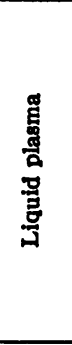 } & ్ㅗㅇ్ㅁำ & $*$ & $?$ & 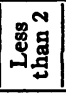 & รี & 욤웜 & $z$ & $\Rightarrow$ & \begin{tabular}{|c|} 
\\
\\
0 \\
0
\end{tabular} & $\underset{m}{\infty}$ & 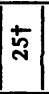 & 偐 & + & $\overrightarrow{\vec{i}}$ & 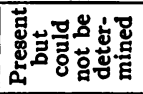 & 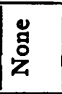 & غٌ & & \\
\hline & & 루워ำ & $\infty$ & & $\infty$ & กุ & 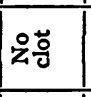 & $\infty$ & $m$ & \begin{tabular}{|l|}
8 \\
0 \\
-
\end{tabular} & & 总 & 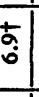 & 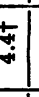 & 索 & t్ర్త & $m$ & 峁 & & \\
\hline & & 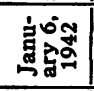 & $N$ & & $\approx$ & ชุ & 品 & ले & 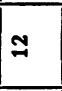 & | & & \pm & $\begin{array}{c} \\
\vdots \\
0 \\
0\end{array}$ & 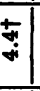 & 节 & 呄 & in & 递 & & \\
\hline & & คั & -1 & & i & & & P & $\cong$ & \begin{tabular}{|l|}
\multirow{2}{*}{} \\
\end{tabular} & & 声 & 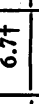 & 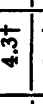 & స్ & 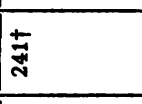 & & 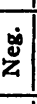 & & 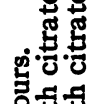 \\
\hline & & ดั้ & 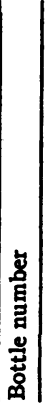 & & 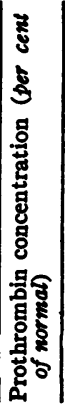 & 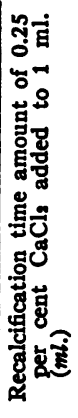 & 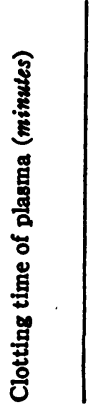 & 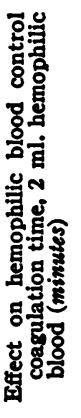 & 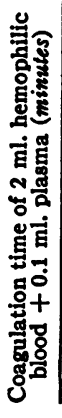 & 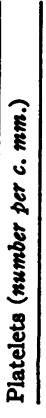 & 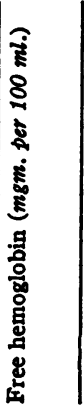 & 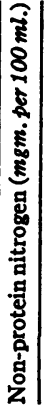 & 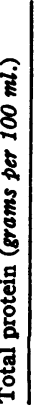 & 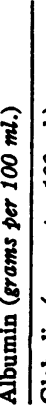 & 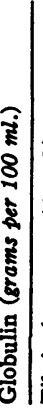 & 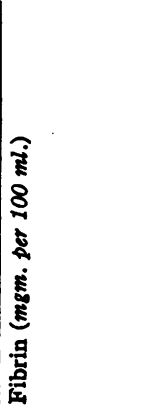 & 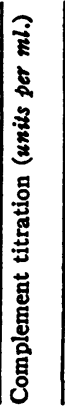 & 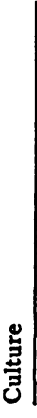 & 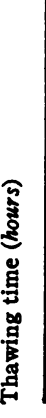 & 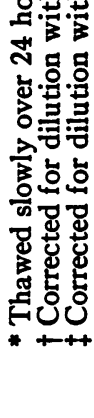 \\
\hline
\end{tabular}




\section{Recalcification time}

As a test of the existence of a complete coagulation mechanism, samples of liquid and frozen plasma were recalcified. The liquid plasma was incoagulable in 2 to 6 months while the frozen plasma retained its clotting ability after the addition of calcium.

\section{Complement}

The data on complement titration indicate that this factor was well preserved in frozen plasma. In liquid plasma, it was lost within the 6 months' period of study.

\section{Miscellaneous}

The $\mathrm{pH}$ of both liquid and frozen plasma rose slightly from a normal value of 7.4 to approximately 8 over the 6 months' period. Counts of refractile bodies resembling platelets ranged from 4000 to 42,000 per c. $\mathrm{mm}$. A decrease in the number was noted in some pools as time went on. This decrease was slightly more marked in the frozen than in the liquid plasma. Free hemoglobin never exceeded $10 \mathrm{mgm}$. per $100 \mathrm{ml}$. in any of the samples submitted to us. Cultures for bacterial growth were uniformly negative.

\section{Rate of destruction of prothrombin}

It would appear from the foregoing studies that the principal blood coagulation component which disappeared from liquid plasma was prothrombin. It seemed advisable, therefore, to study conditions under which the component could be stabilized.

Samples of whole blood were obtained and kept.chilled until arrival in the laboratory. The cells were removed by centrifuging in a refrigerated centrifuge. Samples of plasma were removed under sterile conditions. One set of samples was kept in the icebox, the second set allowed to stand at room conditions. Samples of plasma were removed from each at daily intervals and tested for their prothrombin content and recalcification time. The results are shown in Figure 1.

It is evident that the prothrombin decreased very rapidly in the plasma kept at room temperature, as is well known. Actually, under the conditions of this experiment, in 10 days, there was virtually no prothrombin remaining, and at

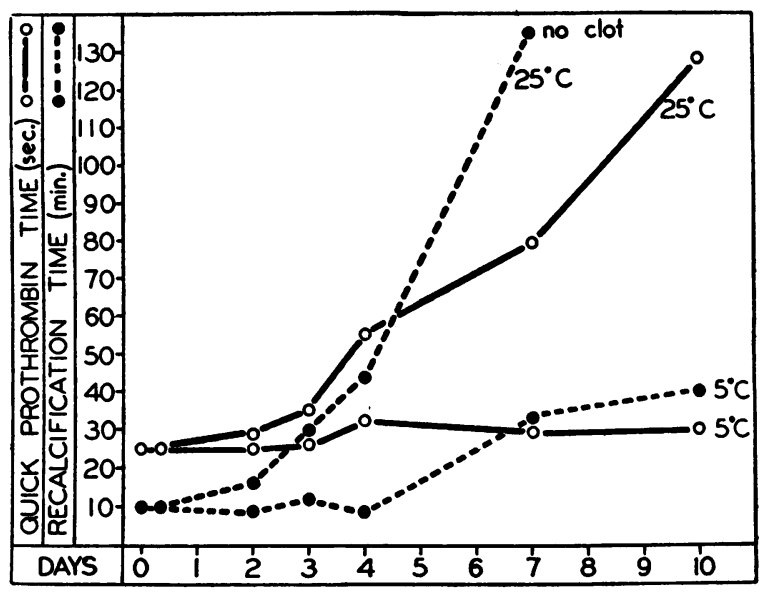

Fig. 1. The Effect of Temperature on the Stability OF PROTHROMBIN

the end of 7 days, the plasma was incoagulable on recalcification. On the other hand, plasma remaining in the icebox lost its prothrombin much more slowly, and the plasma still clotted in 40 minutes at the end of 10 days.

From the increase in recalcification time in the icebox experiment, it would appear that destruction of prothrombin is not the only factor responsible for the loss of the property of coagulability. It has been our experience that changes in recalcification time of normal plasma seldom occur unless the prothrombin is reduced below 10 per cent of normal. In the experiment reported here, the prothrombin concentration remained at 45 per cent of its normal value and yet the recalcification time of the plasma kept in the icebox rose from 10 minutes to $\mathbf{4 0}$ minutes.

While the actual determinations shown in the figure were all made after adjustment to $\mathrm{pH} 7.4$, nevertheless the $\mathrm{pH}$ of the plasma kept in the icebox had in 10 days risen to 8.7, and that at room temperature, to 9.0 .

\section{DISCUSSION}

Lozner and Newhouser (18), in the preceding paper, report that liquid plasma may be administered with minimal untoward reactions, even when preserved at room temperature for over a year. Furthermore, it was observed, from a statistical analysis of the reactions encountered that they became more infrequent as the plasma was aged. The relative ease and 
the low cost of manufacture of liquid plasma and the infrequency of reactions demand a complete appraisal of this therapeutic agent.

The data in the paper immediately following (19) indicate that even after storage in the liquid form for as long as two years, there is minimal protein degradation. It is of interest therefore that the present studies indicate that the physiological activity of prothrombin, and possibly other components of the blood coagulation reaction together with complement, have completely vanished within 6 months. Frozen plasma on the other hand retains such activity.

In evaluating these results with regard to liquid plasma as a therapeutic agent, several considerations are involved. These relate, first of all, to the indications for which the plasma is to be administered and, secondly, to those factors in the plasma which might be concerned with such indications. By far the leading indications for plasma administration are the prevention and treatment of shock resulting from plasma loss following trauma or burns, and the prevention and treatment of hypoproteinemia of various origins. The factors in the plasma concerned with these indications are, primarily, those concerned with the maintenance of colloid osmotic pressure and, secondarily, those concerned with nutrition, coagulation, and immunity. The composition of liquid plasma is adequate for the first indication. With regard to the latter three factors, the present publication deals only with in vitro studies. Certainly these are sufficiently clear-cut to warrant the statement that in patients with hypoprothrombinemia, hemorrhagic diathesis, or infections, the use of stored liquid plasma could not be expected to have any specific therapeutic effect, due to absence of prothrombin, coagulation effect, and complement. The present tendency toward large dosage in burns and trauma may have a significant influence in the final decision on the disadvantage of the use of liquid plasma. One cannot deny that there is a theoretical hazard in administering large amounts of plasma, devoid of coagulation properties and complementary activity. The answer as to whether this hazard is more theoretical than real will depend on extensive clinical trial and investigation under conditions where these properties are required.
Similarly, the nutritional value of such plasma must await long-term balance studies.

\section{SUMMARY}

1. A study of the stability of certain labile components of plasma preserved in the liquid and frozen state has been made.

2. The protein components of both liquid and frozen plasma are well preserved.

3. The coagulation factors of liquid plasma are lost but better preserved in frozen plasma.

4. Complement is lost from liquid plasma but preserved in frozen plasma.

5. The effect of temperature on the preservation of prothrombin is indicated.

The authors acknowledge with gratitude the interest and advice of Doctor George R. Minot during the development of this investigation.

\section{BIBLIOGRAPHY}

1. Newhouser, L. R., and Kendrick, D. B., Human plasma and serum, development and clinical indications. U. S. Nav. M. Bull., 1941, 39, 506.

2. Strumia, M. M., and McGraw, J. J., The development of plasma preparations for transfusions. Ann. Int. Med., 1941, 15, 80.

3. Mahoney, E. B., Kingsley, H. D., and Howland, J. W., The therapeutic value of preserved blood plasma. A summary of one hundred and ten cases. Ann. Surg., 1941, 113, 969.

4. Pohle, F. J., The blood platelet count in relation to the menstrual cycle in normal women. Am. J. M. Sc., 1939, 197, 40.

5. Quick, A. J., The nature of the bleeding in jaundice. J. A. M. A., 1938, 110, 1658.

6. Quick, A. J., Stanley-Brown, M., and, Bancroft, F. W., A study of the coagulation defect in hemophilia and in jaundice. Am. J. M. Sc., 1935, 190, 501.

7. Souter, A. W., and Kark, R., Quick's prothrombin test simplified by the use of a stable thromboplastin. Am. J. M. Sc., 1940, 200, 603.

8. Lozner, E. L., and Taylor, F. H. L., The effect of foreign surfaces on blood coagulation. J. Clin. Invest., 1942, 21, 241.

9. Lee R. I., and White, P. D., Clinical study of the coagulation time of blood. Am. J. M. Sc., 1913, $145,495$.

10. Bing, F. C., and Baker, R. W., The determination of hemoglobin in minute amounts of blood by Wu's method. J. Biol. Chem., 1931, 92, 589.

11. Bing, F. C., Purification of benzidine, and an improved reagent for estimating hemoglobin in blood. J. Biol. Chem., 1932, 95, 387.

12. Shen, S. C., and Ham, T. H., Studies on destruction of red blood cells. 3. Mechanism and complications 
of hemoglobinuria in patients with thermal burns: spherocytosis and increased osmotic fragility of erythrocytes. New England J. Med., 1943, 229, 701.

13. Folin, O., Laboratory Manual of Biological Chemistry. D. Appleton-Century Company, New York, 1934, 5th ed.

14. Howe, P. E., The determination of proteins in blood. A micro method. J. Biol. Chem., 1921, 49, 109.

15. Cullen, G. E., and Van Slyke, D. D., Determination of the fibrin, globulin, and albumin nitrogen of blood plasma. J. Biol. Chem., 1920, 41, 587.

16. Taylor, F. H. L., Lozner, E. L., and Adams, M. A.,
The thrombic activity of a globulin fraction derived from rabbit plasma. Am. J. M. Sc., 1941, 202, 585.

17. Howell, W. H., Hemophilia. Bull. New York Acad. Med., 1939, 15, 3.

18. Lozner, E. L., and Newhouser, L. R., Preservation of normal human plasma in the liquid state. I. A statistical study of 1751 administrations. J. Clin. Invest., 1944, 23, 343.

19. Lozner, E. L., et al., Preservation of normal human plasma in the liquid state. III. Studies on chemical and physico-chemical changes during the second year of storage. J. Clin. Invest., 1944, 23, 357. 